\title{
Effect of vitamin D supplementation on cold restraint induced oxidative stress in rats
}

\author{
Khaled Abd-Elsater Elewa ${ }^{1}$, Misbahuddin Mohd Rafeeq ${ }^{2 \star}$ and Mohammad Mohiuddin ${ }^{1}$ \\ ${ }^{1}$ Department of Physiology, Rabigh College of Medicine, King Abdulaziz University, Jeddah 80200, \\ Kingdom of Saudi Arabia. \\ ${ }^{2}$ Department of Pharmacology, Rabigh College of Medicine, King Abdulaziz University, Jeddah 80200, \\ Kingdom of Saudi Arabia.
}

Accepted 23 May, 2012

\begin{abstract}
Free radicals are electrically charged molecules that are produced during the course of normal metabolism. They spontaneously attack lipids, proteins, nucleic acids and other biologically important molecules. Polyunsaturated lipids are particularly sensitive to their attack. Free radicals and oxidative stress have been reported to be involved in tissue injury associated with a number of diseases. The present study was done to investigate the effect of vitamin $D$ on cold induced oxidative stress in rats. Rats were divided into control, acute and chronic stress groups with and without vitamin D treatment. Tissue total antioxidant concentration and tissue lipid peroxide were measured in all groups. Statistical analysis was done using the computer software program prism version 3.3. Result showed that the acute cold restraint decreased the total antioxidant levels below the control but there was a significant increase in antioxidant levels of the group in which vitamin $D$ is administered. Chronic cold restraint produces non significant decrease of total antioxidant levels. There was a significant increase of total antioxidant level in chronic cold restraint rats treated vitamin $\mathrm{D}$. Moreover, the chronic cold group with vitamin D produced significant increase of plasma total antioxidant levels above control, acute, chronic groups. The lipid peroxide levels were increased significantly in acute cold restraint group with and without vitamin $D$ as compared to control. Vitamin D produces non significant decrease in lipid peroxide levels in acute and chronic cold restraint group.
\end{abstract}

Key words: Vitamin D, stress, defense mechanism, animal experimentation.

\section{INTRODUCTION}

Free radicals are electrically charged molecules that are produced during the course of normal metabolism. The superoxide radical appear to play a central role since others are formed from it. The main source for formation of superoxide is through activation of nicotinamide adenine dinucleotide phosphate oxidase enzyme (NADPH), which is located on the outer side of plasma membrane. This activation leads to initiation of the respiratory burst with the generation of superoxide anion

${ }^{\star}$ Corresponding author. E-mail: drmisbah98@gmail.com. Tel: +966550309636 .
(Kelly et al., 2010).

Free radicals spontaneously attack lipids, proteins, nucleic acids and other biologically important molecules (Davydov and Shvets, 2001). Free radicals may abstract an atom of hydrogen from polyunsaturated fatty acids and initiate the chain reaction known as lipid peroxidation. Polyunsaturated lipids are particularly sensitive to free radical attack because they contain methylene groups in interrupted double bond systems whose hydrogen atoms are susceptible to abstraction (Varashree and Bhat, 2011).

An imbalance between free radical generation and various antioxidant defense systems leading to the accumulation of free radicals is called oxidative stress. Free 
Table 1. Effects of acute and chronic cold stress on total antioxidant levels ( $\mathrm{mmol} / \mathrm{L})$ and lipid peroxide (mean \pm $\mathrm{SE})$ as compared with control non-stressed group.

\begin{tabular}{|c|c|c|}
\hline Parameter & Total antioxidant (mean $\pm \mathrm{SE}$ ) & Lipid peroxide (mean \pm SE) \\
\hline Control & $5.01 \pm 0.68^{\#_{\star}}$ & $0.03 \pm 0.004^{\#_{\star}}$ \\
\hline Acute cold restraint & $2.00 \pm 0.93^{\#^{0}}$ & $0.17 \pm 0.007^{\#^{\circ}}$ \\
\hline Acute cold restraint with vitamin D & $2.94 \pm 0.82^{\#^{2}}$ & $0.13 \pm 0.003^{\#^{0}}$ \\
\hline Chronic cold restraint & $4.96 \pm 0.55^{\star}$ & $0.05 \pm 0.01^{*}$ \\
\hline Chronic cold restraint with vitamin D & $5.39 \pm 0.6^{*}$ & $0.02 \pm 0.002^{*}$ \\
\hline
\end{tabular}

\#, Significant $(<0.05)$; *, highly significant $(<0.001)$.

Free radicals and oxidative stress have been reported to be involved in tissue injury associated with a number of diseases including rheumatoid arthritis, atherosclerosis, immunological disorders, cataract, diabetes mellitus, cancer, hepatic diseases, psoriasis, ischemia-reperfusion injury of the heart, brain, pancreas, and others (Abd Ellah, 2011).

The present study was done to investigate the effect of vitamin $\mathrm{D}$ on cold induced oxidative stress in rats to elucidate the role of vitamin $\mathrm{D}$ as an antioxidant.

\section{MATERIALS AND METHODS}

\section{Animal model}

Sprague-Dawley adult male albino rats 90 in number (from Assiut university animal house) weighing 200 to $250 \mathrm{~g}$ at the beginning of the experiment were used. The rats were housed individually under standard conditions of temperature and humidity and a 12-h light/dark cycle (lights on at 08:00 a.m.) with free access to food and water. All animals were maintained under constant conditions for 14 days before stress. All experiments were performed during the same time of day, between 9 am and 3 pm to avoid variations due to diurnal rhythms (Noriyoshi et al., 2000). All of the procedures regarding the care and use of animals and animal experimentation in this study were complied with the Guide for the Care and Use of Laboratory Animals published by the US National Institutes of Health.

\section{Animal groupings/treatment}

The rats were divided into three groups: a) control, b) acute cold restraint and c) chronic cold restraint. Rats in control group were left in animal house without exposure to any form of stress. Acute cold restraint was induced by fixing the four limbs to a wooden board and placing it in a refrigerator at $4^{\circ} \mathrm{C}$ for six hours one time while chronic cold restraint was induced by placing the board once daily for 9 days (Zaidi et al., 2005). Rats in acute and chronic stress groups were divided into further subgroups including: 1) Acute stress with and without vitamin treatment; and 2) chronic stress with and without vitamin treatment. Vitamin $D(15 \mathrm{mg} / \mathrm{kg}$ of body weight) was administered orally prior to and after $6 \mathrm{~h}$ of restraint stress exposure one time in acute groups and once daily for 9 days in chronic groups. Control, acute and chronic stress groups without treatment were given distilled water (DW) orally ( $3 \mathrm{ml} / \mathrm{kg} \mathrm{bw})$ for the same duration accordingly (Zaidi and Banu, 2004).

\section{Collection of samples}

Animals were killed immediately after the last session of immobilization (still in the restrainer) using sodium pentobarbital. A weighed portion of the liver and brain were put in a test tube containing $5 \mathrm{ml}$ of acidified butanol. The mixture was homogenized in a conical tube immersed in ice. The homogenate was centrifuged at $1000 \mathrm{rpm}$ for $5 \mathrm{~min}$. Then $2.5 \mathrm{ml}$ of the supernatant was transferred to a test tube and used for measurements (Zaidi et al., 2005).

\section{Biochemical measurements}

\section{Determination of tissue total antioxidant concentration}

Total antioxidant concentration was assayed by spectrophotometer. $1 \mathrm{ml}$ of the chromogen was added to $20 \mu \mathrm{l}$ of double deionized water to form the reagent blank, and $1 \mathrm{ml}$ of chromogen to the $20 \mu \mathrm{l}$ of standard, to prepare the standard. Then samples were measured after addition of one $\mathrm{ml}$ of chromogen to $20 \mu \mathrm{l}$ of the sample. Initial absorbance was measured. After that, $200 \mu \mathrm{l}$ of diluted substrate was added to each sample, standard and blank. Mixture was measured after exactly $3 \mathrm{~min}$.

\section{Determination of tissue lipid peroxides}

The total amount of lipid peroxides was assayed by the thiobarbituric acid method described by Okhawa et al. (1979). This measures the malondialdehyde equivalent substances, which are breakdown products of lipid peroxides.

\section{Statistical analysis}

Statistical analysis was done using the computer software program prism (Comshare's version of a decision support system = DSS) version 3.3. Data are expressed as mean \pm SEM. Statistical significance for data was determined using a one-way analysis of variance (ANOVA) with Bonferroni's Multiple Comparison Test to find inter-group significance. $\mathrm{P}<0.05$ was considered statistically significant and $<0.001$ highly significant.

\section{RESULTS}

Table 1 represents the mean \pm SE values of total antioxidant and lipid peroxide levels of all groups. Result 
show that the acute cold restraint decreased the total antioxidant levels below the control but there is a significant increase in antioxidant levels of the group in which vitamin $\mathrm{D}$ is administered as compared to control. On the other hand, chronic cold restraint produces non significant decrease of total antioxidant levels below control levels. There is a significant increase of total antioxidant level in chronic cold restraint rats treated vitamin $\mathrm{D}$ compared to chronic cold restraint rats not given Vitamin D. Moreover, the chronic cold with vitamin D produces significant increase of plasma total antioxidant levels above control, acute, chronic groups. Regarding the lipid peroxide levels, results indicate that the lipid peroxide levels are increased significantly in acute cold restraint group with and without vitamin D as compared to control. Vitamin D produces non significant decrease in lipid peroxide levels in acute cold restraint group. On the other hand, vitamin D does not reduce the lipid peroxide levels significantly in chronic cold restraint group.

\section{DISCUSSION}

The obtained data in the present work clearly demonstrated that the total antioxidant levels significantly decline in acute cold restraint stress group as compared with control group with insignificant changes in the chronic stress groups. These results were previously confirmed by Zaidi et al. (2005) and Hussain et al. (2009).

These results could be explained on the basis that acute stress hormones (glucocorticoids and catecholamines) produce metabolic changes in the enzyme activities of glucose and phosphate pathways (Vaz et al., 2011). Thus, the decreased blood antioxidant levels were due to the decreased glucose 6-phosphate dehydrogenase activity which caused the diminished production of NADPH (glucose-6-phosphate dehydrogenase regenerates NADPH) and in consequence also of antioxidant enzymes (Dede et al., 2002). Another possible mechanism is that, under normal circumstances, formation of $\mathrm{O}_{2}$ is kept under control of antioxidants. In acute stress, the production of $\mathrm{O}_{2}$ is increased and total antioxidants are decreased if this production is at a rate that overwhelms the capacity of the endogenous antioxidant defense system to remove them. The result of such imbalance results in $\mathrm{O}_{2}$ mediated damage (Dede et al., 2002). The use of vitamins $D$ therapy not only helps in stress adaptation but also protect against the direct injurious effects of oxidants (Concepción et al., 2004; Keong et al., 2006).

In the present study, acute cold restraint stress groups produce a significant increase in lipid peroxides levels as compared with control group. This result agrees with Dede et al. (2002), Zaidi et al. (2005) and Hussain et al. (2009). Significant increase of lipid peroxide indicates the generation of reactive oxygen species as a result of acute stress. It may be due to one or more of the metabolic pathways that are known to produce free radicals which include: 1) the xanthine $(X)$ and xanthine oxidase (XO) system; 2) the cyclooxygenase pathway of the arachidonic acid metabolic system; 3 ) the electron transport system of mitochondria; 4) the activated neutrophil system; and 5) the amyloid protein system (Sung et al., 2000). Cold alone causes the release of reactive oxygen species in cell membrane, thus leading to the production of lipid peroxide (Dede et al., 2002). The increase of free radicals during acute stress is associated with a decrease in mitochondrial respiratory control, loss of sarcoplasmic reticulum (SR)/endoplasmic reticulum (ER) integrity, and increased levels of peroxidation products and lipid peroxidation (Concepción et al., 2004).

The results of the present work showed that the lipid peroxides levels in chronic cold restraint stress group were insignificantly increased. This result was previously confirmed by McEwen (2007) who reported that cessation of stress or chronic stress returns the body to its original equilibrium. Supplementation with vitamin $D$ decreased $\mathrm{O}_{2}{ }^{-}$and increased total antioxidant status. These findings confirm the antioxidant properties of vitamins $D$ and indicate that treatment influences generation of oxygen free radicals and improves antioxidant defenses. Processes contributing to these effects are probably related to the direct scavenging actions of vitamins $D$ (Keong et al., 2006). Another possible mechanism in the vitamin-treated rats is that the activation of NADPH oxidase is decreased and activity of superoxide dismutase is increased. Because NADPH oxidase is the major source of superoxide anion in vascular cells, decreased activation of the enzyme would result in reduced generation of the oxygen free radical. On the other hand, enhanced activation of superoxide dismutase would lead to increased dismutation of $\mathrm{O}_{2}^{-}$, which further decreases $\mathrm{O}_{2}{ }^{-}$concentration (Zaidi and Banu, 2004).

\section{Conclusion}

The supplementation of vitamin D increases the body's ability to fight the stress related free radical generation. More studies are needed with larger sample size and more parameters.

\section{REFERENCES}

Abd Ellah M (2011). The role of liver biopsy in detection of hepatic oxidative stress. Vet. Med. Int. 613602 doi 10.4061/2011/613602

Concepción S, Scott J, Thaler D, Folstein M, Martin A (2004). Decreased levels of plasma vitamin D and increased concentrations of inflammatory and oxidative stress markers after stroke. Stroke, 35: 163-166.

Davydov V, Shvets N (2001). Lipid peroxidation in the heart of adult and old rats during immobilization stress. Exp. Gerontol. 36(7):1155-1160. 
Dede S, Deger Y, Meral I (2002). Effect of short-term hypothermia on lipid peroxidation and antioxidant enzyme activity in rats. J. Vet. Med., 49(A): 1-3.

Hussain Z, Amresh G, Singh S, Rao C (2009). Antidiarrheal and antiulcer activity of Amaranthus spinosus in experimental animals. Pharmaceut. Biol. 47(10):932-939.

Kelly E, Wang L, Ivashkiv L (2010). Calcium-activated pathways and oxidative burst mediate zymosan-induced signaling and IL-10 production in human macrophages. J. Immunol. 15:184(10):55455552.

Keong C, Singh H, Singh R (2006). Effects of palm vitamin E supplementation on exercise-induced oxidative stress and endurance performance in the heat. J. Sports Sci. Med. 5:629-639.

McEwen BS (2007). Physiology and Neurobiology of Stress and Adaptation: Central Role of the Brain. Physiol Rev. 87(3):873-904.

Noriyoshi S, Masaru Y, Takemi G, Yoshimitsu A (2000). Stimulation of prostaglandin $\mathrm{E} 2$ and interleukin-1B production from periodontal ligament cells of old rat subjected to mechanical stress. J. Gerontol. 55:B489-B495.

Okhawa H, Ohishi N, Yagi K (1979). Assay for lipid peroxides in animal tissues by thiobarbituric acid reaction. Anal Biochem. 95:351-358.
Sung N, Dayong W, Bradley S, Guohua C, Simin N (2000). Effect of long-term dietary antioxidant supplementation on influenza virus infection. J. Gerontol. Biol Sci. 55:B496- B503.

Varashree BS, Bhat GP (2011). Correlation of Lipid Peroxidation with Glycated Haemoglobin Levels in Diabetes Mellitus. Online J. Health Allied Sci. 10(2):11.

Vaz G, Xavier C, Coimbra C, Fontes M, Borges E (2011). Increased jejunal absorption of glucose in rats submitted to blockade of gaba-a receptors in the hypothalamic paraventricular nucleus. Open Neuroendocrinol. 4:120-126.

Zaidi S, Banu N (2004). Antioxidant potential of vitamins A, E and C in modulating oxidative stress in rat brain. Clin. Chem. Acta 340(12):229-233.

Zaidi S, Al-Qirmi, Banu N (2005) Effects of antioxidant vitamins on glutathione depletion and lipid peroxidation induced by restraint stress in the rat liver. Drugs R\&D 6(3):157-165. 